\title{
Effects of Nucleus Initialization on Event-by-Event Observables
}

\author{
B. Mattos Tavares ${ }^{a}$, H.-J. Drescher ${ }^{b}$, and T. Kodama ${ }^{a}$ \\ ${ }^{a}$ Instituto de Física, Universidade Federal do Rio de Janeiro, 68528, 21945-970, Rio de Janeiro, RJ, Brasil \\ ${ }^{b}$ Frankfurt Institute for Advanced Studies (FIAS), Johann Wolfgang Goethe-Universiät, \\ Max-von-Laue-Str. 1, 60438 Frankfurt am Main, Germany
}

\section{Received on 29 September, 2006}

\begin{abstract}
In this work we present a study of the influence of nucleus initializations on the event-by-event elliptic flow coefficient, $v_{2}$. In most Monte-Carlo models, the initial positions of the nucleons in a nucleus are completely uncorrelated, which can lead to very high density regions. In a simple, yet more realistic model where overlapping of the nucleons is avoided, fluctuations in the initial conditions are reduced. However, $v_{2}$ distributions are not very sensitive to the initialization choice.
\end{abstract}

Keywords: Elliptic flow; Fluctuations in the initial conditions; Hydrodynamics

\section{INTRODUCTION}

The main goal of studying ultra-relativistic heavy ion reactions is to identify (or not) the formation of the Quark-Gluon Plasma (QGP). Event-by-event (EbyE) analysis of global observables can significantly contribute in this direction [1]. One way to measure EbyE fluctuations is the statistical approach: each observable in an event should contain fluctuations and the distribution of such an observable can be characterized by its mean value and higher moments [2].

The most interesting fluctuations are dynamical, since they give important information about the formation of the system. Possible examples are the occurrence of jets, giving rise to fluctuations in the high $p_{t}$ tail of transverse momentum distributions, and the fluctuation in the anisotropic flow coefficient $v_{2}$ due to unusual hard/soft equation of state or fluctuating initial conditions $[1,3]$.

Other sources of fluctuations of statistical or technical nature can also be present in EbyE distributions. For example, finite multiplicity affects the determination of $\left\langle p_{t}\right\rangle$, ratios of multiplicities of particle species and also the strength of the anisotropic flow coefficient, due to imprecise determination of event plane [1]. In this work, we are interested in a technical source of fluctuations, commonly present in event generators, the initialization of the positions of the nucleons in a nucleus before the collision.

\section{HYDRODYNAMICAL MODEL}

We have proposed that dynamical and technical sources of fluctuations can be studied in the framework of relativistic hydrodynamics, which allows one to separate the relevant physics from statistical noise [4]. If the local thermal equilibrium is attained in relativistic heavy ion collisions, hydrodynamical description may be most adequate for the space-time evolution of the system. Once the initial conditions (spatial configuration of 4-velocity field and conserved currents) of the system are specified, the principal factor which characterizes the hydrodynamical motion is the equation of state of the matter. When the hydrodynamical prescription is not valid anymore, one must employ a decoupling criterion to generate particles, i.e, hadrons. So we have only three inputs in the hydrodynamical approach: initial conditions, equation of state and the decoupling criterion.

In this work, we employ the numerical code SPHERIO [5], which solves the hydrodynamical equations of motion in 3 dimensions and can deal with any kind of spatial configuration in the initial conditions. For the equation of state, we have adopted a quark-gluon free gas with a bag constant of $380 \mathrm{MeV} / \mathrm{fm}^{3}$ for the QGP phase, and a hadron resonance gas with excluded volume (only for baryons) in the confined phase. Almost all the resonances up to $2.5 \mathrm{GeV}$ have been used. The phase boundary is obtained via the Gibbs criterion, and the transition is first order for every chemical potential. As a decoupling criterion, we adopted Cooper-Frye procedure [6] with a fixed freeze-out temperature of $140 \mathrm{MeV}$. For further details in the equation of state and decoupling prescription used, see [7].

\section{NUCLEUS INITIALIZATION: CORRELATED VERSUS UNCORRELATED NUCLEON POSITIONS.}

For the initial condition, we use the NEXUS event generator [9]. Based on the Gribov-Regge model of hadronic collisions, it generates a spatial distribution of the energymomentum tensor $T^{\mu \nu}$ and the baryon number density $n_{B}$ on the hyper-surface $\tau=$ const. Monte-Carlo generation of events from this model give rise to physical fluctuations in the initial conditions. However, the procedure chosen to initialize the nuclei before the collision, may generate also unphysical fluctuations, which can influence EbyE observables.

Usually one employs the Wood-Saxon distribution

$$
\rho(r)=\rho_{0} \frac{1}{1+\exp [(r-R) / D]},
$$

to determine the initial position of the $A$ nucleons within a nucleus. The constants in eq. (1) are the nuclear density $\rho_{0}=$ $0.16 \mathrm{fm}^{-3}$, the nucleus radius $R$ and the diffuseness parameter $\mathrm{D} \sim 0.55 \mathrm{fm}$ (usually).

The nucleons determined this way are completely uncorrelated, like a gas of free particles. So, it can happen that several nucleons occupy the same position in space, which is un- 
physical. However, this method is used in many Monte-Carlo codes, since it is widely believed that unphysical fluctuations average out, at least for inclusive spectra. Some models, e.g HIJING [8] are aware of this problem, and employ a rejection technique for nucleons which are closer to each other than a given distance. However, for realistic distances (in the order of 2 times the proton radius $\sim 1.6 \mathrm{fm}$ ) this leads to a pushing of nucleon positions towards the shell and lowers density in the center. Nexus generates nuclei, with completely uncorelated positions.

The most suitable initialization of a nucleus would be a selection of nucleon positions according to a realistic wave function which includes correlations [10]. Each nucleon would be surrounded by a corelation hole [10], which prevents other nucleons from occupying the same space. For simplicity, we employ a lattice model in order to demonstrate the effect. The nucleons are placed on a body-centered cubic lattice (BCC) and the positions are then accepted with a probability $\rho / \rho_{0}$ given by eq. (1). The packing efficiency of a BCC lattice is $e=8 / 3 * \pi /(4 / \sqrt{3})^{3}=0.68$ and the lattice spacing is $l=\left(e / \rho_{0} / \pi * 3 / 4\right)^{1 / 3} * 4 / \sqrt{3}=2.32 \mathrm{fm}$ [11]. This method correctly reproduces the density in the center of the nucleus, as can be seen in Fig. 1. To avoid lattice artifacts, each nucleus is rotated by some random angles. We shall call this method lattice (or correlated) initialization.

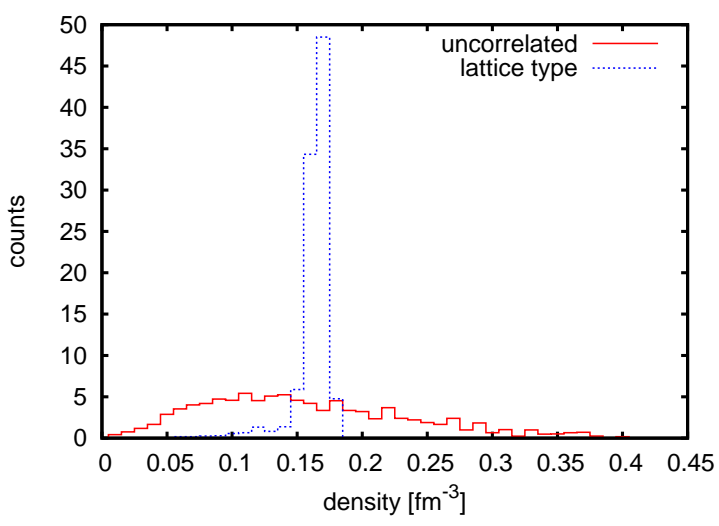

FIG. 1: Distribution of the nucleon density in the center of the nucleus $(r<4 \mathrm{fm})$, for correlated (dashed line) and uncorrelated (solid line) nucleons.

In Fig. 2 we show a plot for the energy density distribution for the correlated (dashed line) and the uncorrelated (full line) cases. Each distribution represents 100 NEXUS events for Gold-Gold collisions with $\sqrt{s}=200 \mathrm{GeV}$ per nucleon pair, with zero impact parameter $b$ (central collisions). One can easily check that the energy density from correlated nucleons is narrower than the uncorrelated case, as expected. It can also be seen that the average energy density is a larger for the lattice type initialization.

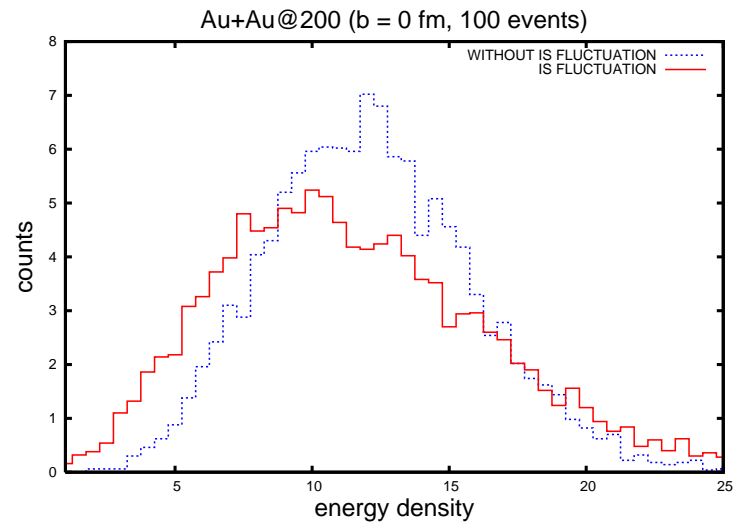

FIG. 2: Energy density distribution for central collisions $(b=0)$.

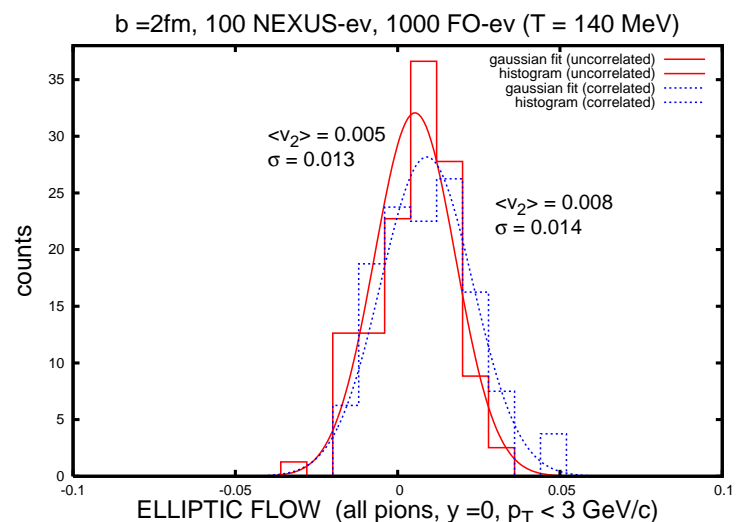

FIG. 3: Elliptic flow distributions for central collisions ( $b=2 \mathrm{fm}$ ).

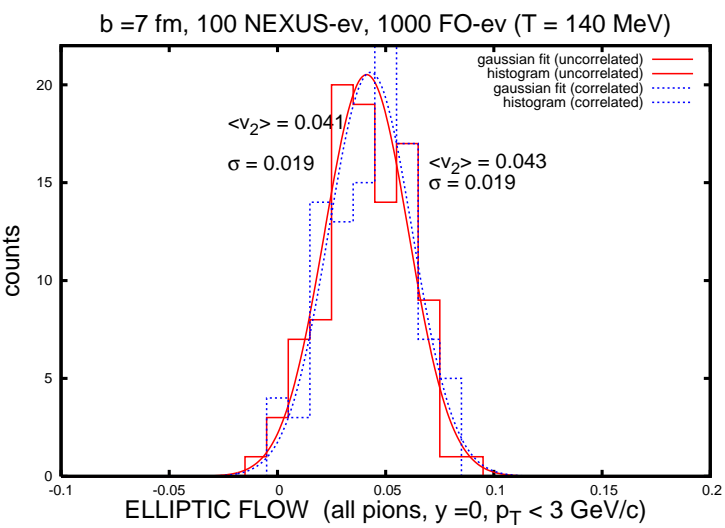

FIG. 4: Elliptic flow distributions for mid-central collisions $(b=7$ fm).

\section{RESULTS}

In Figs. 3, 4 and 5 we show EbyE distributions for the elliptic flow coefficient $v_{2}$ (integrated over $p_{t}$ ) of pions, computed for $b=2 \mathrm{fm}$ (central collisions), $b=7 \mathrm{fm}$ (mid-central) and $b=$ 


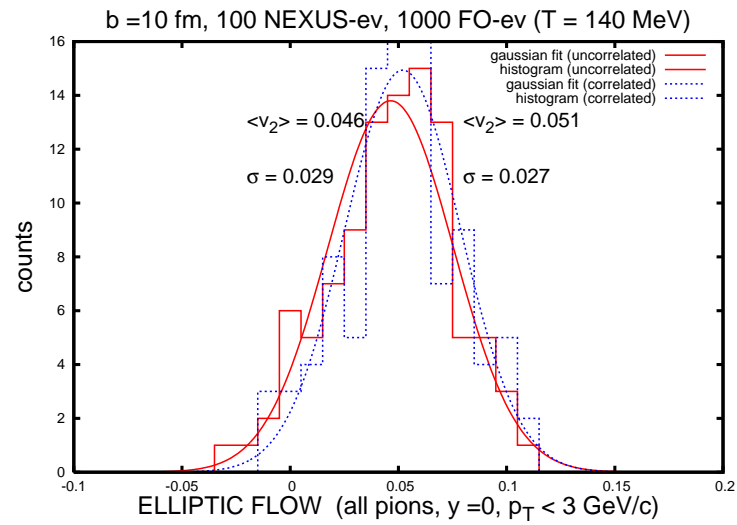

FIG. 5: Elliptic flow distributions for peripheral collisions $(b=10$ $\mathrm{fm})$.

$10 \mathrm{fm}$ (peripheral collisions), at mid-rapidity, for Gold-Gold reactions at $\sqrt{s}=200 \mathrm{GeV}$ per nucleon pair. We show the histograms and the Gaussian fits for the observable originated from the correlated (dashed) and uncorrelated (solid) nucleus initialization. We computed 100 events per type of initializa- tion at each impact parameter. Despite the differences in the energy density distributions, the $v_{2}$ in all centralities shows very similar variances $\sigma$. That may be caused by the hydrodynamical expansion which reduces the effects of the fluctuations.

\section{CONCLUSIONS AND PERSPECTIVES}

In this work we studied the effects of nucleus initialization on EbyE observables. The $v_{2}$ parameter is not very sensitive to the choice of nucleus initialization. The influence on other observables are in progress. We also plan to study the effects of impact parameter distribution, reaction plane determination (for the $v_{2}$ case) and other finite multiplicity related observables. The goal is to develop a method that separates physical from statistical fluctuations.

\section{Acknowledgments}

the authors would like to thanks for discussions with A. Dumitru. This work has been done with the support of FAPESP, CNPq, DAAD and CAPES.
[1] S. Voloshin, V. Koch, and H. G. Ritter, Phys. Rev. C 60, 0224901 (1999).

[2] See for example S. Jeon, V. Koch, Phys. Rev. Lett. 85, 2076 (2000).

[3] Y. Hama, T. Kodama, and O. Socolowski, Braz. J. Phys. 35, 24 (2005).

[4] B. Mattos Tavares, PhD thesis.

[5] for SPH procedure in RHIC see C. E. Aguiar, et al J. of Phys. G 27, 75 (2001).

[6] F. Cooper, G. Frye, Phys. Rev. D 10, 186 (1974); F. Cooper, G. Frye, and E. Schonberg, Phys, Rev. D 11, 192 (1975).

[7] C. E. Aguiar, Y. Hama T. Kodama, and T. Osada, Nucl. Phys.
A 698, 639c (2002); C. E. Aguiar, Y. Hama, T. Kodama, and T. Osada, in New States of hadronic interactions - PASI 2002, AIP Conference Proceedings, 631, 686 (2002).

[8] M. Gyulassy, X.-N. Wang Comp. Phys. Comm. 83, 307 (1994).

[9] H.J. Drescher et al, Phys. Rev. C 65, 054902 (2002).

[10] G. Baym, B. Blätel, L. L. Frankfurt, H. Heiselberg, and M. Strikman Phys. Rev. C 52, 1604 (1995). C. K. Wong, Phys. Rev. C 35, 545 (1987).

[11] C. Kittel, introduction to Solid State Physics, John Wiley \& Sons (1996). 